\title{
Compaction of RNA Hairpins and their Kissing Complexes in Native Electrospray Mass Spectrometry
}

\author{
Josephine Abi-Ghanem ${ }^{1}$, Clémence Rabin ${ }^{1}$, Massimiliano Porrini ${ }^{1}$, \\ Frédéric Rosu $^{2}$, Valérie Gabelica ${ }^{1 *}$ \\ ${ }^{1}$ Univ. Bordeaux, CNRS, INSERM, ARNA, UMR 5320, U1212, IECB, F-33600 Bordeaux, \\ France. \\ ${ }^{2}$ Univ. Bordeaux, CNRS, INSERM, IECB, UMS 3033, F-33600 Pessac, France. \\ * Corresponding author: v.gabelica@iecb.u-bordeaux.fr
}

\begin{abstract}
:
When electrosprayed from typical native MS solution conditions, RNA hairpins and kissing complexes acquire charge states at which they get significantly more compact in the gas phase than their initial structure in solution. Here we also show the limits of using force field molecular dynamics to interpret the gas-phase structures of nucleic acid complexes in the gas phase, as the predicted CCS distributions do not fully match the experimental ones. We suggest that higherlevel calculation levels should be used in the future.
\end{abstract}




\section{Introduction:}

Ion mobility spectrometry (IMS) separates ions according to charge and shape, based on their electrophoretic mobility in a drift tube filled with a buffer gas such as helium. ${ }^{1-2}$ The measured physical quantity is the collision cross section ( ${ }^{\exp } \mathrm{CCS}$ ), and the interpretation of this structural information requires theoretical calculations $\left({ }^{\text {calc }} \mathrm{CCS}\right)$ on ion structures in vacuo. Upon native electrospray ionization, intact molecules as well as noncovalent complexes can be preserved. However during the transfer in gas phase, structural rearrangements may occur. ${ }^{3-8}$ Experimental and theoretical approaches initially suggested that Watson-Crick hydrogen bonds and stacking are preserved, ${ }^{9-10}$ but we recently showed that DNA and RNA duplexes in the gas phase with low charge states undergo a significant compaction ( $>20 \%$ ) compared to the solution structures. ${ }^{11}$ This raises the concern that other higher-order DNA or RNA structures containing double helix motifs may also end up more compact in the gas phase than they were in solution. DNA hairpins, pseudoknots and cruciforms were studied by ion mobility before, ${ }^{12}$ but these experiments were carried out in $5 \mathrm{mM}$ aqueous $\mathrm{NH}_{4} \mathrm{OAc}$. At this lower ionic strength, higher charge states are produced, and consequently the structures are less compact. Also the molecular dynamics trajectories at the time were 2 ns. Here we tested RNA structures sprayed from aqueous solutions at physiological ionic strength $\left(150 \mathrm{mM} \mathrm{NH}_{4} \mathrm{OAc}\right)$, i.e. conditions mimicking the biologically relevant ionic strength, ${ }^{13}$ and performed more extensive force field molecular dynamics calculations.

Here, we studied RNA hairpins and RNA-RNA kissing complexes structures (Figure 1) in the gas phase. Hairpins (also called "stem-loops") are formed by a duplex part (the stem) and a loop of unpaired nucleotides. Their flexibility depends on their composition and on the size of the stem and loop. The hairpin is a frequent motif in natural RNA folding and interactions. ${ }^{14}$ For example, the tRNA structure involves three hairpins. ${ }^{15}$ Also, hairpins with tetranuleotide loops are naturally present in a variety of RNAs such as rRNAs, group I introns or ribozymes. ${ }^{16-17}$ RNA-RNA kissing complexes are formed when the nucleotides in the loops of two RNA hairpins are complementary. The smallest loop-loop interaction is formed by only two nucleotides in each loop, ${ }^{18}$ but usually involve more nucleotides. Here we studied the RNAI:RNAII kissing complex involved in the replication of the ColE1 plasmid in Escherichia coli (actually their inverse sequences RNAIi and RNAIIi, which form a more stable complex, often used for model biophysical studies), ${ }^{19-20}$ and the 
binding of TAR RNA sequence from the mRNA of the HIV-1 virus with the R06 aptamer (itself a hairpin), designed to bind TAR and prevent the binding to the TAT protein. ${ }^{21}$ An NMR structure of RNAIi:RNAIIi (PDB: 2BL2) ${ }^{22}$ and an X-ray crystal structure of TAR:R06 (PDB: 1JLT) ${ }^{23}$ have been published, and will be used as starting points to examining the differences between gas-phase and solution structures.

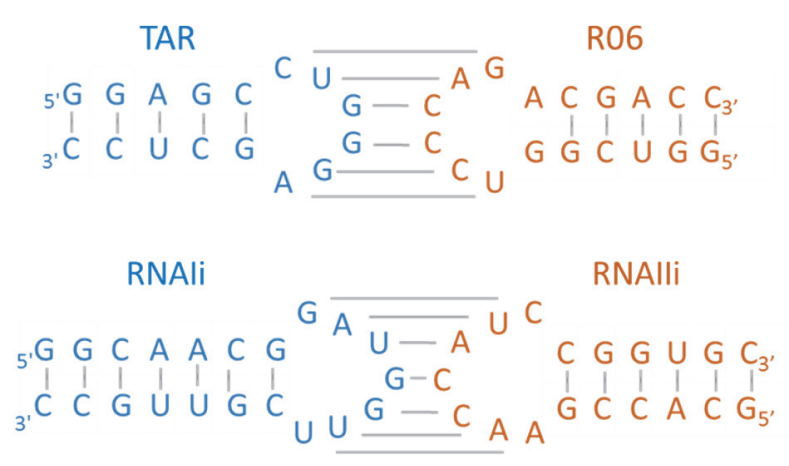

Figure 1: Sequences and secondary/tertiary structures of the TAR:R06 and RNAIi:RNAIIi kissing complexes.

\section{Experimental:}

\section{Oligonucleotides}

Lyophilized oligonucleotides were purchased from Integrated DNA Technology (Leuven, Belgium) with standard desalting. They were dissolved in RNAse-free water (Ambion, Fisher Scientific, Illkirch, France), then a stock solution at $100 \mu \mathrm{M}$ of each hairpin was prepared in 150 $\mathrm{mM} \mathrm{NH}_{4} \mathrm{OAc}$ (itself prepared from BioUltra $\approx 5 \mathrm{M}$ stock solution, Fluka, Sigma-Aldrich, SaintQuentin Fallavier, France). This stock was desalted using Amicon Ultra-0.5 3K ultrafiltration devices (Merck Millipore, Saint-Quentin en Yvelines, France). The individual hairpins were then heated at $90^{\circ} \mathrm{C}$ for $90 \mathrm{~s}$ and then quickly cooled on ice to obtain the hairpin structure. The injected solutions were $10 \mu \mathrm{M}$ of each hairpin in $150 \mathrm{mM} \mathrm{NH}_{4} \mathrm{OAc} .2 \mu \mathrm{M}$ of $\mathrm{dT}_{6}$ was added as a reference for quantitation. The kissing complexes were prepared by mixing equimolar amounts of hairpins.

\section{Native Electrospray Ionization Mass Spectrometry (ESI-MS)}

Experiments were performed on an Agilent 6560 DTIMS-Q-TOF instrument (Agilent Technologies, Santa Clara, CA), with the dual-ESI source operated in negative ion mode. The 
syringe pump flow rate was $180 \mu \mathrm{L} / \mathrm{h}$. The drift tube was filled with helium (temperature $=296 \pm$ $1 \mathrm{~K})$. The pressure in the drift tube was $3.89 \pm 0.01$ Torr, and the pressure in the trapping funnel was $3.67 \pm 0.01$ Torr (this ensures that only helium is present in the drift tube). In our default conditions, the source temperature and fragmentor voltage were set at $200^{\circ} \mathrm{C}$ and $350 \mathrm{~V}$, and the Trap Entrance Grid Delta was 4 V (for an explanation of these parameters, see reference 23). Other parameters of a lesser influence on ion internal energy were: trap fill time $=1 \mathrm{~ms}$, trap release time $=100 \mu \mathrm{s}$, high pressure funnel RF $=180 \mathrm{~V}$, trap funnel delta $=160 \mathrm{~V}$, trap funnel $\mathrm{RF}=190 \mathrm{~V}$, trap entrance grid low $=102 \mathrm{~V}$, trap entrance $=101 \mathrm{~V}$, trap exit $=99 \mathrm{~V}$, trap exit grid 1 low $=95 \mathrm{~V}$, trap exit grid 1 delta $=6 \mathrm{~V}$, trap exit grid 2 low $=94 \mathrm{~V}$, trap exit grid 2 delta $=10 \mathrm{~V}$. These parameters and their tuning have been discussed extensively in a previous publication. ${ }^{24} \mathrm{We}$ found that maintaining the source block temperature at $200{ }^{\circ} \mathrm{C}$ prevented the contamination of the helium drift gas, ${ }^{24}$ and tests with ubiquitin ${ }^{7+}$ and a fragile G-quadruplex reassured us that changing this temperature from $50^{\circ} \mathrm{C}$ to $220^{\circ} \mathrm{C}$ was not affecting significantly the internal energy of ions. Note that with such source conditions, the RNA ions are not entirely stripped from all their ammonium adducts (see supporting information Figures S1-S2).

The collision cross section distributions were reconstructed for each species by summing the IMMS signals over the entire ammonium adduct distribution. To produce more activating conditions, the fragmentor voltage was set to $600 \mathrm{~V}$. Alternatively, we also increased the trap entrance grid delta to $12 \mathrm{~V}$, resulting in similar outcomes (not shown herein). The procedure used to reconstruct the CCS distributions is discussed in detail elsewhere. ${ }^{25}$ Briefly, we first determined the CCS of the peak center by the step-field method, which involves measuring the arrival time as a function of the drift voltage (which was stepped from $390 \mathrm{~V}$ to $790 \mathrm{~V}$ ). The CCS is determined from the slope of the linear regression using

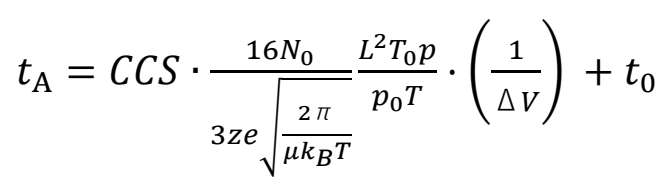

The drift tube length is $L=78.1 \mathrm{~cm}, T=297 \pm 1 \mathrm{~K}$, the pressure is measured by a capacitance gauge ( $p=3.89 \pm 0.01$ Torr), $N_{0}=2.687 \times 10^{25} \mathrm{~m}^{-3}$ at $p_{0}=760$ Torr and $T_{0}=273.15 \mathrm{~K}, z$ the ion net charge, $\mu$ is the reduced mass of the ion-gas pair (the gas being helium), $e$ is the electron charge and $k_{\mathrm{B}}$ is the Boltzmann constant. Then the arrival time distribution obtained at the lowest drift 
voltage $(390 \mathrm{~V})$ is converted to a CCS distribution using

$C C S=a \cdot \frac{z}{\sqrt{\mu}} \times t_{A}$

Where the factor $a$ is determined from the $\left(t_{\mathrm{A}}, C C S\right)$ values obtained for the peak center.

\section{MD in solution and representative structures}

The MDs in solution were performed using as starting point the PDB coordinates $2 \mathrm{JLT}^{23}$ for TAR/R06 and 1BJ2 for RNAi/RNAii. ${ }^{22}$ The structures were minimized and equilibrated using the module pmemd.MPI of Amber $15^{26}$ suite of packages using the parmbsc 0 force field with $\chi$ OL 3 correction. ${ }^{27-29}$ The complexes were neutralized with $\mathrm{Na}^{+}$ions and hydrated with TIP3P water molecules in a truncated octahedron. ${ }^{30-31}$ Periodic boundary conditions at constant temperature $(300 \mathrm{~K})$ and pressure $\left(1\right.$ bar) using the Berendsen algorithm were applied. ${ }^{32}$ Covalent bonds involving hydrogens were restrained using SHAKE, ${ }^{33}$ with a 2-fs integration time-step. Longrange electrostatic interactions were treated using the particle mesh Ewald (PME) ${ }^{26}$ algorithm with a radial cutoff of $9 \AA$; the same cut-off was used for the van der Waals interactions. The nonbonded pair-list was updated heuristically and the center-of-mass motion removed every 10 ps. Water molecules and counter-ions were energy-minimized (steepest descent followed by conjugate gradient) and equilibrated at $100 \mathrm{~K}$ for $100 \mathrm{ps}$ at constant volume and temperature while RNA molecules were positionally restrained. The whole system was then heated from $100 \mathrm{~K}$ to $300 \mathrm{~K}$ over $10 \mathrm{ps}$ by steps of $5 \mathrm{~K}$ with harmonic positional restraints on solute atoms (5.0 $\mathrm{kcal} / \mathrm{mol} / \AA^{2}$ force constant). The simulation was continued at constant pressure and temperature. The positional restraints were gradually removed over $250 \mathrm{ps.}$

MD production runs of 300 ns to $1 \mu$ s were started from the end point of the equilibration. For the 16-mer hairpin TAR and the 18-mer hairpin R06, a multiple molecular dynamic (MMD) approach was used. From the end point of the equilibration, 25 trajectories of $10 \mathrm{~ns}$ each (total $250 \mathrm{~ns}$ ) were launched with new velocities for each trajectory. The cpptraj ${ }^{34}$ module of AmberTools 15 was used to calculate the radius of gyration and the representative structure. The statistical analysis was carried out using the R package. ${ }^{35}$ Image rendering was performed with tachyon in VMD. ${ }^{36}$ 


\section{Localized charges}

After the representative structure in solution was chosen, some phosphate groups have to be neutralized by protons. To choose which ones, we applied an in-house python script to generate 1000 structures with random protonation schemes. Using Amber module sander we calculated a single point energy for each of these structures (no geometry optimization in the gas phase at this stage). The 200 structures showing the lowest energy were chosen and their protonation scheme was displayed as histograms, to deduce the most probable locations (further information below in the results). We then placed the protons on the representative structure according to those locations.

\section{MD in gas phase}

The whole system was heated from $100 \mathrm{~K}$ to $300 \mathrm{~K}$ during $100 \mathrm{ps}$, and 50 independent attempts were compared (further discussion in the results section). Then gas phase MD simulations were run with the sander module of Amber15, in canonical ensemble (at $\mathrm{T}=298 \mathrm{~K}$ ) using a Langevin thermostat, implementing a direct Coulomb summation for evaluating the electrostatic interactions, using an integration time-step of $1 \mathrm{fs}$ and no radial cut-off for the non-bonded forces. Because the screening effect of the solvent is missing in vacuo, we set no radial cut-off for both Coulomb and van der Waals interactions and the PME algorithm cannot be applied. In order to calculate the CCS from the gas phase MD simulations, snapshots were collected every 50 ps. Theoretical CCS values were calculated with EHSSrot algorithm ${ }^{37}$ with the parameterization of Siu et al., ${ }^{38}$ which accuracy was validated previously on rigid nucleic acid structures (Gquadruplex, G-duplex) of similar size..$^{39-40}$

\section{DFT calculations}

DFT calculations were run on TAR $^{4-}$ were performed using Gaussian09 ${ }^{41}$ (M06-2X functional, ${ }^{42}$ Grimme D3 dispersion correction ${ }^{43}$ and 6-31G(d,p) basis-set). A geometry optimization was first carried out on the truncated hairpin TAR $14^{3-}$ lacking the terminal base pair, because the optimization could not start converging when starting from the full 16-mer hairpin. Then, the terminal base pair was added, one more phosphate group was deprotonated, and the entire 16-mer $\mathrm{TAR}^{4-}$ could be geometry optimized. The electrostatic potentials were calculated from the total electronic density. 


\section{$\underline{\text { Results and Discussion }}$}

Representative electrospray mass spectra in soft conditions are shown in supporting Figure S1. In $150 \mathrm{mM} \mathrm{NH}_{4} \mathrm{OAc}$, the hairpins dominate, and the kissing complexes are minor. Although $150 \mathrm{mM}$ corresponds to a physiological concentration of monovalent salts, ${ }^{13}$ the presence of magnesium would be required to promote kissing complex formation in vitro and in vivo. However, adding magnesium to the ESI-MS conditions is detrimental to the signal-to-noise ratio, and thus harsher in-source conditions have to be used to distinguish the $\mathrm{Mg}^{2+}$ adducts, ${ }^{44}$ which would be detrimental to the study of gas-phase structures as they emerge from the electrospray process. Here we focus on assessing the gas-phase structures produced by electrospray in source conditions just hard enough to desolvate and partially decluster the RNA polyanions from their ammonium counterions. An example of result obtained in the presence of $\mathrm{Mg}^{2+}$ ions will be shown as well, in the supporting information.

\section{RNA hairpins and kissing complexes are significantly more compact in the gas phase than in solution}

The experimental collision cross sections in helium are determined from the arrival time distributions. The CCS values of the peak centers in repeated measurements ( $n=3$ minimum) were within $1 \%$. The standard relative uncertainty on the collision cross section of the peak center, estimated by propagation of uncertainty on temperature, pressure, tube length and slope of the $t_{\mathrm{a}}=\mathrm{f}(1 / \Delta V)$ linear regression is estimated at $\sim 1.2 \%$. The full width at half maximum of the CCS distributions was calculated as reported previously. ${ }^{25}$ Even after correction for instrumental broadening by diffusion, the peaks are broad, meaning that the width of the CCS distribution is due to gas-phase ensemble containing multiple conformations. The values are reported in Table 1 and representative experimental distributions are shown in Figure 2.

To calculate the CCS values corresponding to the structures in solution, we used the coordinates from the published structures of the kissing complexes. The hairpins were extracted from their respective kissing complexes. The calculated collision cross sections in helium of these starting structures are given in Table 1. The ion mobility measurements were performed in two sets of conditions: soft conditions wherein some ammonium adducts remain (see Supporting Figure S2 
for the adduct distribution) and the CCS was determined from the entire ammonium adduct distribution (in black on Figure 2), and harsh conditions where ammonium ions were stripped and the CCS was determined from ions with no adduct (in red on Figure 2). Even in soft conditions, the CCS values are already $\sim 20$ to $30 \%$ smaller in the gas phase than in the starting solution structures. The entire experimental CCS distribution is below the expected one in solution. Using harsher conditions compacts the kissing complexes even further away from the solution structures.

The compaction can occur at various stages of electrospray: after acquisition of the final charge state in droplets but with solvent and counter-ions still around, after desolvation but while counterions are still around, or after full desolvation and declustering. Note that we currently ignore when charging and desolvation occur, respectively. For nucleic acids, however, we know from the $\Delta m$ of adduct loss that water is removed at an earlier stage than ammonium counter-ions. On oligonucleotides, it is in our experience impossible to obtain a good signal-to-noise with water adducts unambiguously preserved; the peaks would be lost in the noise. The fact that, even with ammonium adducts around, the gas-phase structures are already more compact that in solution indicates that the electrospray process (charging, desolvation, or both) is already affecting the structure. The same compaction happens to RNA double helices. Supporting Information Figure S3 shows the CCS distributions of control duplexes having the same composition as the kissing complexes. The CCS distributions overlap. Hence the CCS distributions are also useless to distinguish double helix from kissing complex structures.

Given that, like most RNA complexes, kissing complex formation is favored by the presence of magnesium, we also attempted to record ion mobility data in presence of $200 \mu \mathrm{M} \mathrm{Mg}(\mathrm{OAc})_{2}$ in soft conditions. However, this experiment failed for RNAIi:RNAIIi and gave poor signal-to-noise ratio for TAR:R06. The TAR:R06 results (Supporting Information Figure S4) nevertheless show that collision cross sections in soft conditions are similar with 0,1 or $2 \mathrm{Mg}^{2+}$ adducts. The collision cross section distribution for the entire adduct distribution (including ammonium and magnesium adducts) is centered at $1017 \AA^{2}$, i.e. close to the one recorded in only $\mathrm{NH}_{4} \mathrm{OAc}\left(986 \AA^{2}\right)$ and already more compact than the starting solution structure $\left(1219 \AA^{2}\right)$. In harsher conditions, all these structures get even more compact, but we note that the complex with $2 \mathrm{Mg}^{2+}$ is compacting a bit less than the with 0 or $1 \mathrm{Mg}^{2+}$ adducts. This recalls the offset collision-induced unfolding of proteins in presence of divalent cations. ${ }^{45-46}$ 
To summarize, together with our previous study on DNA and RNA double helices, ${ }^{11}$ our results show that, at the charge states naturally produced from aqueous $\mathrm{NH}_{4} \mathrm{OAc}$ mimicking physiological ionic strength, double stranded regions in nucleic acids undergo compaction upon electrospray.
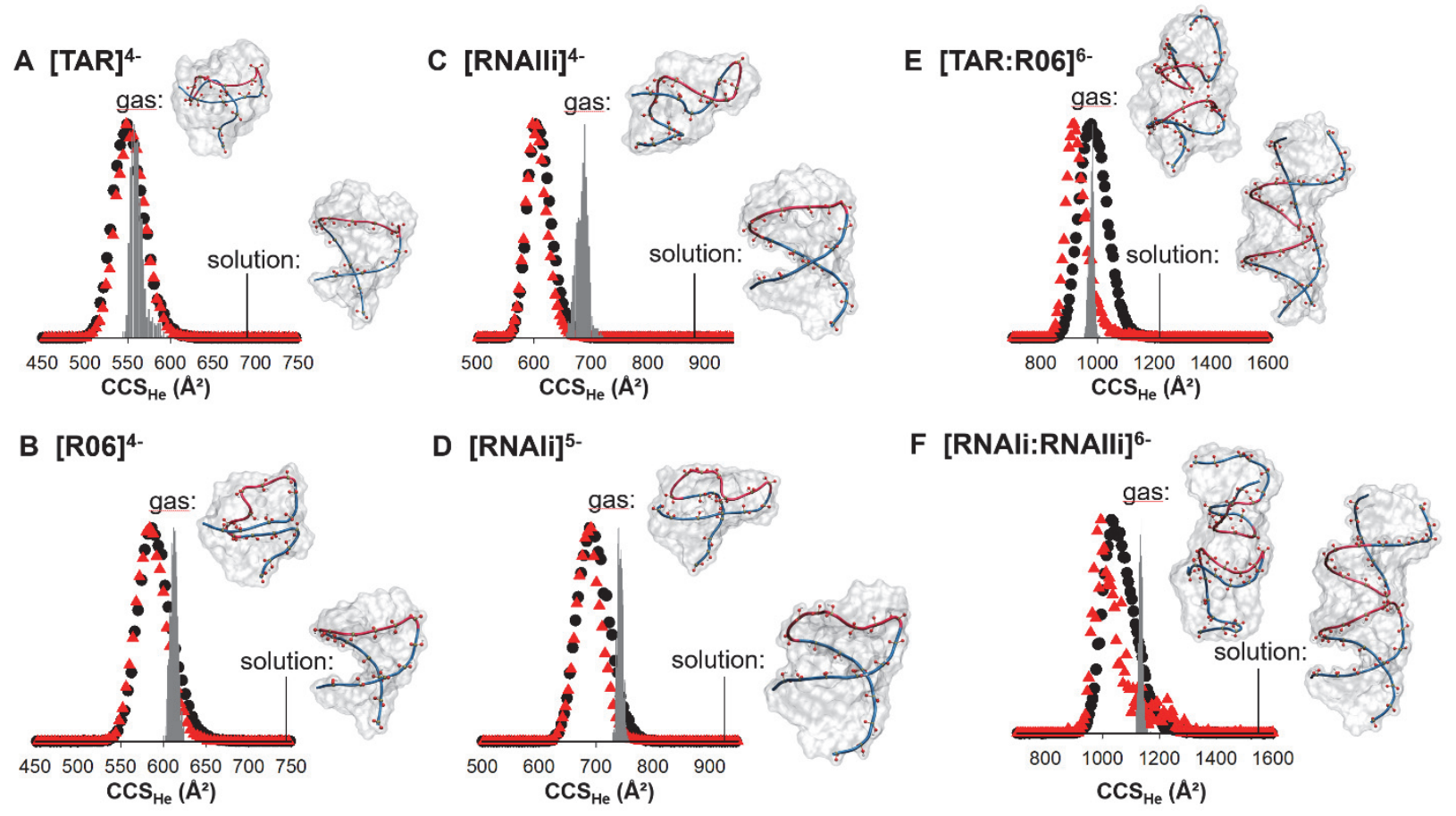

Figure 2: Experimental gas-phase collision cross section distributions for the hairpins (A-D) and kissing complexes $(\mathrm{E}, \mathrm{F})$. The effect of pre-ion mobility activation is seen by comparing black distributions (fragmentor at $350 \mathrm{~V}$ ) and red curves (fragmentor at $600 \mathrm{~V}$ ). 


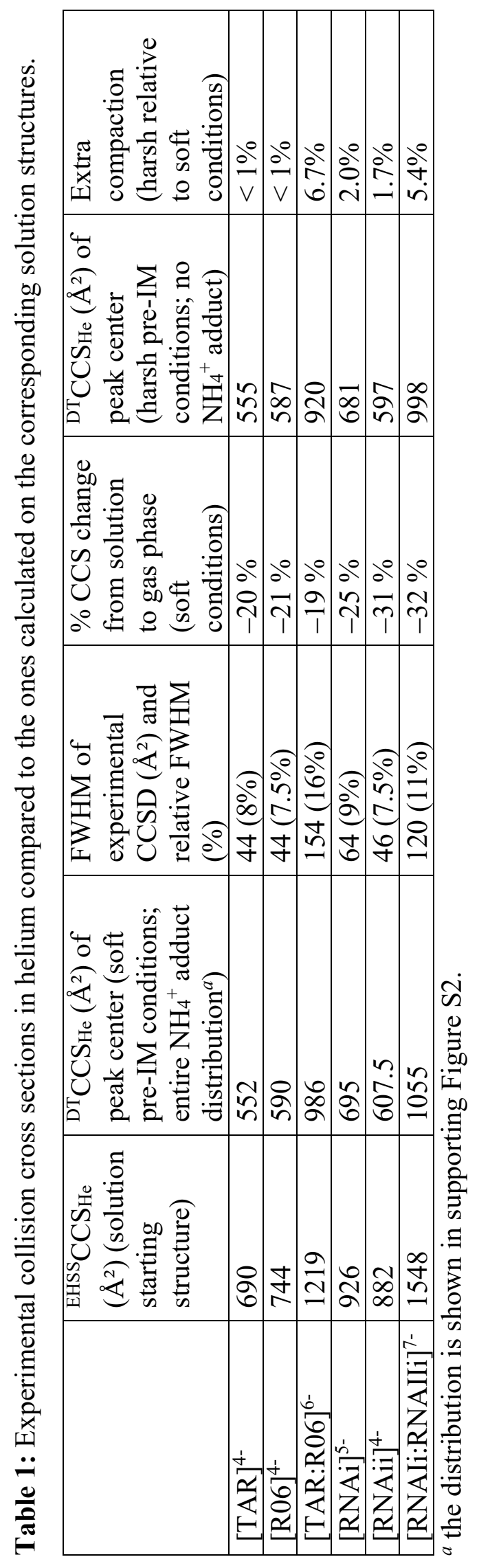




\section{Can molecular dynamics (MD) with molecular mechanics force field account for the gas- phase compaction?}

The parmbsc 0 force field with $\chi \mathrm{OL} 3$ correction is appropriate to model RNA structures in solution. Here we will test whether this force field is appropriate to account for the rearrangements that occur in the gas phase. The general approach is to (1) generate solution structures (either by taking published coordinates or by running short solution dynamics starting from them), then (2) strip the structures of their solvent and reduce the number of charges to match the ones observed by ESI-MS, then (3) prepare the system for gas-phase dynamics and finally (4) run them long enough or at high temperature enough for the experimentally relevant rearrangements to occur. At each step, choices have to be made. We will show here that only making choices that maximize compaction at each step that the force field MD simulations can render a compaction that approaches the experimentally observed one, and that even with the bias of insight for the choice of modeling parameters the extent of modeled compaction is in most cases still not enough.

In the first step, we explored whether hairpins and kissing complexes might adopt a wider variety of conformations in solution, by running solution force field MD simulations starting from the published coordinates. To sample a variety of conformations we ran multiple molecular dynamic (MMD) starting from the experimental structure and running 25 trajectories for $10 \mathrm{~ns}$ each. The kissing complexes are stable systems in solution and MD simulation were done for $300 \mathrm{~ns}$. A first clustering was based on the radius of gyration, as its calculation is easier to implement on-the-fly, and the correlation of $r_{\mathrm{g}}$ with CCS, although not perfect, ${ }^{47}$ suffices to capture starting points which are the most compacted conformation (low $r_{\mathrm{g}}$ ), and compared the results with less compact starting points (high $r_{\mathrm{g}}$ ). Although the visual differences are minor, as we will show below this choice influences the final results.

Next, we tested different protonation schemes. Once the representative structure is selected, the choice is whether to localize the charge (LC) or use a distributed charge model (DC). ${ }^{48}$ With the DC model (taking TAR as an example), the net charge of all the 15 phosphate groups is reduced and the total charge of the hairpin will be of -4 and distributed equally along the phosphate group of the RNA. With the LC model, assuming that only the backbone phosphate groups can be protonated, one needs to add 11 protons out of the 15 phosphate groups with 1365 different ways of positioning them, for a given conformation. Here we randomly protonate the hairpin 1000 times. 
A single point energy calculation with a classical force field was used to classify the resulting structures (Supplementary Figure S5). Among the 200 structures with the lowest energy, we observed the sites that are the most often deprotonated, to define the most probable (MP) protonation. The least probable (LP) localized charge distribution was obtained by filtering the 200 structures with the highest energy.

The next step is the heating of the molecular system up to $300 \mathrm{~K}$ to prepare it for the MD in the gas phase. Note that this section describes the heating process that prepares the system for the actual (300 ns to $1 \mu \mathrm{s}) \mathrm{MD}$ runs at $300 \mathrm{~K}$. We repeated the heating process and observed different outcomes, leading to a broad distribution of CCS values (Figure 3). The gas-phase CCS always decreases compared to the solution, already during the heating step. Thus, depending on the heating trajectory, starting points for the gas-phase MD could be closer to or further from the experimental CCS value. We also tested different heating protocols (Table 2). The resulting distributions for $\mathrm{TAR}^{4-}$ (starting from the most compact solution conformation, localized charges, most probable proton distribution) are shown in Figure 3B. The compaction observed from 0 to 50 ps (Figure 3A) continues slightly to longer times. However a slower heating did not lead to particularly more compact conformations (compare long2 and long1). For the remainder of the paper, we settled on the "medium" heating conditions defined in Table 2.

Table 2: Parameters of the four heating processes tested herein. The change of temperature is applied over steps/iterations Istep1 through Istep2.

\begin{tabular}{|l|l|l|l|}
\hline & \multicolumn{2}{|l|}{ From 0 to 300 K } & Total length (ps) \\
\hline & Istep1 (ps) & Istep2 (ps) & \\
\hline Short & 0 & 20 & 50 \\
\hline Medium & 0 & 20 & 100 \\
\hline Long1 & 0 & 20 & 200 \\
\hline Long2 & 0 & 100 & 200 \\
\hline
\end{tabular}



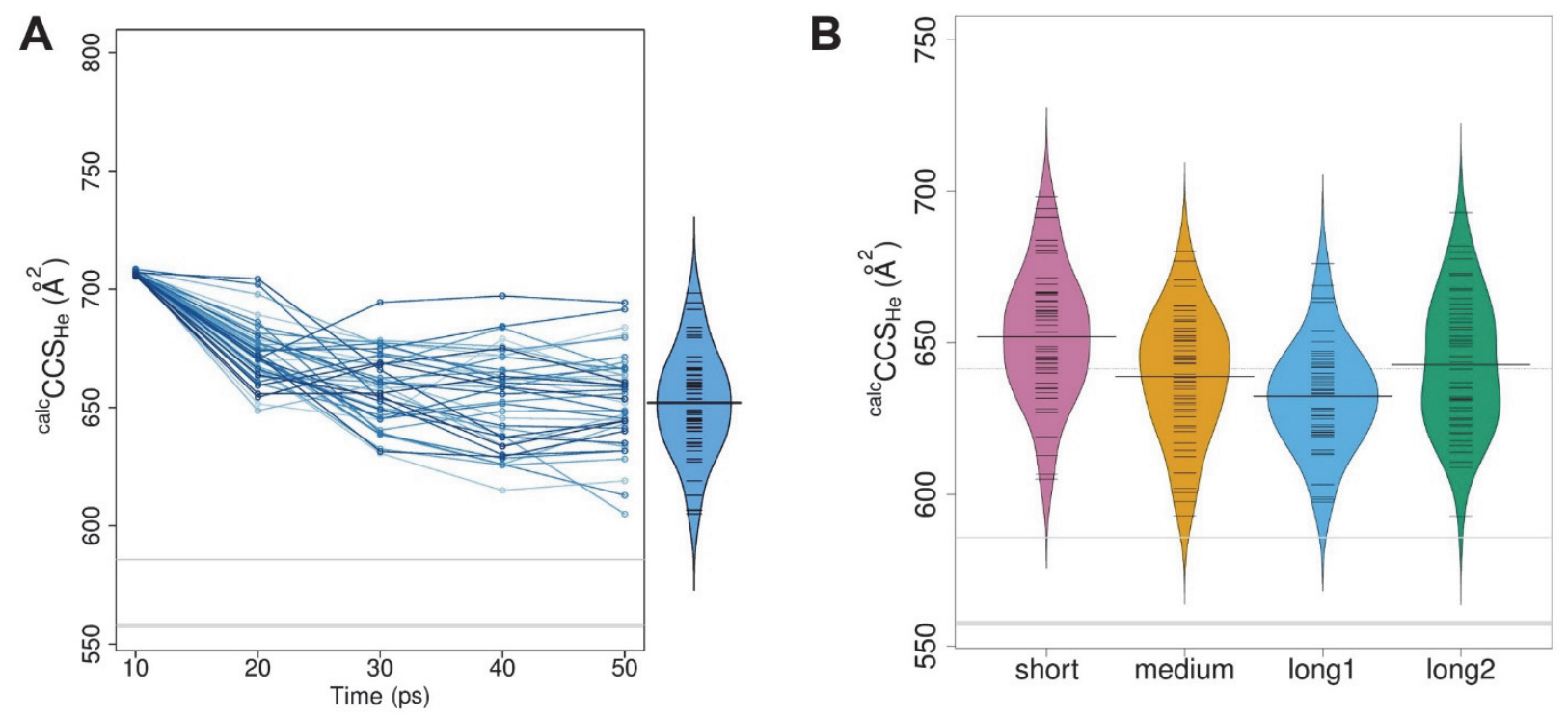

Figure 3: Effect of the of the heating process on the CCS distribution. A) Evolution of the CCS along the 50 ps heating process ( 50 replicates) and final CCS distribution represented as a beanplot. B) CCS distribution following different heating processes (defined in Table 2). The bold grey line indicates the center of the experimental distribution, and a thin grey line its half-maximum on the high-CCS side.

We now compare the distributions of CCS values at the end of the heating process, for the different protonation schemes and with different starting solution structures. Figure 4A shows that, for $\mathrm{TAR}^{4-}$, the MP charge distribution combined with the low starting radius of gyration gives the CCS distribution at the lowest values, i.e. closest to the experimental ones. As expected, the structure with the least probable protonation scheme does not compact as much. The one with distributed charges does not match well with experimental values either. This was initially surprising, because distributed charges were reported to lead to better base-base interaction energies and better DNA double helix preservation in the gas-phase models, ${ }^{48}$ but we note that the structures modeled in that paper were all extended ones. Our results suggest that the localized charges scheme is more relevant for gas-phase RNA at the compact charge states produced in native MS conditions. For $\mathrm{TAR}^{4-}$, the distributions do not significantly depend on the initial structure chosen, but they do for R06 $^{4-}$ (Figure 4B): depending on the starting structure, the conformations converge towards a different ensemble, and starting from a more compact structure converges towards a more compact gas-phase ensemble. 

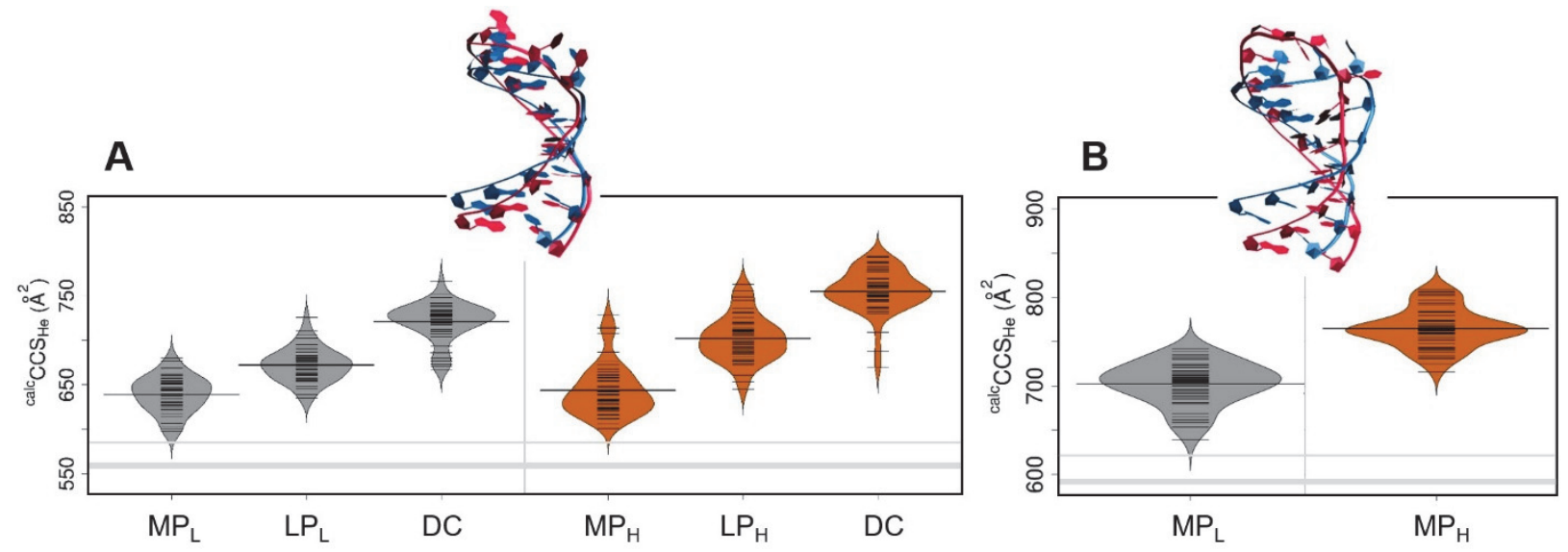

Figure 4: A) Distribution of CCS at the end of the heating process for different protonation schemes of $\mathrm{TAR}^{4-}$ for a low $(10.7 \AA$, grey) or high $(11.7 \AA$, orange) radius of gyration radius starting point: $\mathrm{MP}=$ most probable localized charges, $\mathrm{LP}=$ least probable localized charges, $\mathrm{DC}$ $=$ distributed charges. B) Distribution of CCS at the end of the heating process for R06 $6^{4-}$, for the most probable localized charge distribution applied to a low (11.2 $\AA$, grey) or high (12.9 $\AA$, orange) gyration radius starting point. The structures with low radius of gyration are shown in blue and with high radius of gyration in pink. The bold grey line indicates the center of the experimental distribution, and a thin grey line its half-maximum on the high-CCS side.

Note that after the heating steps, all calculated CCS distributions are still centered at higher values than the experimental ones. During the next step, i.e. long gas-phase MD trajectories, the conformations will have a chance to rearrange into even more compact structures (Figure S6). However, the final ensemble of conformations will depend on the combination of choices made for steps (1)-(3).

The calculated gas-phase CCS histograms shown in Figure 1 were obtained by running gas-phase force field $\mathrm{MD}$ on the most compact gas-phase conformer produced by heating of the most probable localized charge model, itself built from the most compact solution conformer. But even with all these human-imposed biases (because we knew the gas-phase CCS values before doing the modeling), when the hairpins become larger, the mismatch between calculated and experimental CCS increases. An interesting observation nevertheless is that for a compaction to occur in the models, the loop must close on the stem, and this outcome was influenced by the choice of the proton location. 
For the kissing complexes, the workflow described above for hairpins gave even larger discrepancies. The experimentally observed degree of compaction could not be reproduced by the models. In particular, the stems and loops could not come close to one another. We thus applied further restraints during the heating step, in a similar way as described previously for DNA double helices: ${ }^{11}$ protonated and deprotonated phosphate groups from the stem and loop that faced one another were forced to come close to one another during the heating (Figure S7). With that procedure, the models had collision cross sections in the range of the experimentally observed ones. It does not mean that the structures shown in Figure 2 are the gas phase structures, but it means that the gas-phase structures are as compact as those shown.

\section{Discussion: the limitations of force fields to describe electrosprayed nucleic acids}

The general conclusion of the force field molecular dynamics study is that this approach is not viable to interpret the experimental collision cross sections in terms of structure. We also learned that the proton location matters, because the compaction is due to the formation of new (nonnative) hydrogen bonds between phosphate groups. However, the global compactness is generally underestimated by force fields in the gas phase, and this is problematic to interpret ion mobility spectrometry results.

To reveal the underlying reasons, we tested the gas-phase structural remodeling of a biologically relevant hairpin at a much higher level of theory: density functional theory (DFT) with the M06$2 \mathrm{X}$ functional (well adapted to nucleic acids), ${ }^{49}$ and a sufficient basis set $(6-31 \mathrm{G}(\mathrm{d}, \mathrm{p}))$. The largest hairpin for which it could be done is $\mathrm{TAR}^{4-}$. Figure $5 \mathrm{~A}$ shows the starting structure, with the electrostatic potential calculated from the total electron density (preliminary calculations on the shorter hairpin TAR14- are shown in supporting information Figure S8). The charges in the initial structure are localized (deprotonated phosphate groups $n^{\circ} 1,6,12$ and 14 are shown in red on scheme 5B, and clearly appear in red with the color coding representing the electrostatic potential). Figure 5C shows the optimized structure: it has compacted significantly, the base pairs are nevertheless well preserved, and new hydrogen bonds have now formed, notably between neutralized $\mathrm{P}-\mathrm{O}-\mathrm{H}$ groups and charged $\mathrm{P}-\mathrm{O}^{-}$groups. 
In the final structures, the charges are thus more delocalized owing to shared protons (no more obvious red phosphate groups), but starting from and appropriate localized charge distribution was probably necessary for the compaction to happen. The collision cross section of the final (DFToptimized) structure is $592 \AA^{2}$, still on the high side of the experimental one, showing that further compaction occurs in practice. The final structure indeed has many void spaces, and proper conformational exploration would be required, but this is not attainable at this level of theory on our computers.

Scheme 5B shows the new hydrogen bonding pattern. Note that the base pairs are preserved. In addition to the $\mathrm{P}-\mathrm{O}^{-} \cdots \mathrm{H}^{+} \ldots{ }^{-} \mathrm{O}-\mathrm{P}$ ionic hydrogen bonds (red dotted lines), we also observe a pattern of $\mathrm{P}-\mathrm{O}-\mathrm{H} \cdots \mathrm{O}=\mathrm{P}$ hydrogen bonds (blue dotted lines). New hydrogen bonds formed between distant sites for example, the stem and the loop (i.e., phosphates 1 with 9, 2 with 8) are likely those responsible for the greatest compaction. The precise pattern, and thus the extent of compaction and the final structure, will very likely depend on the protonation scheme chosen for modeling. Moreover, we can postulate that protons can be mobile in a hairpin, as shown previously by Car-Parrinello molecular dynamics simulations on 7 -mer single strands. ${ }^{50}$
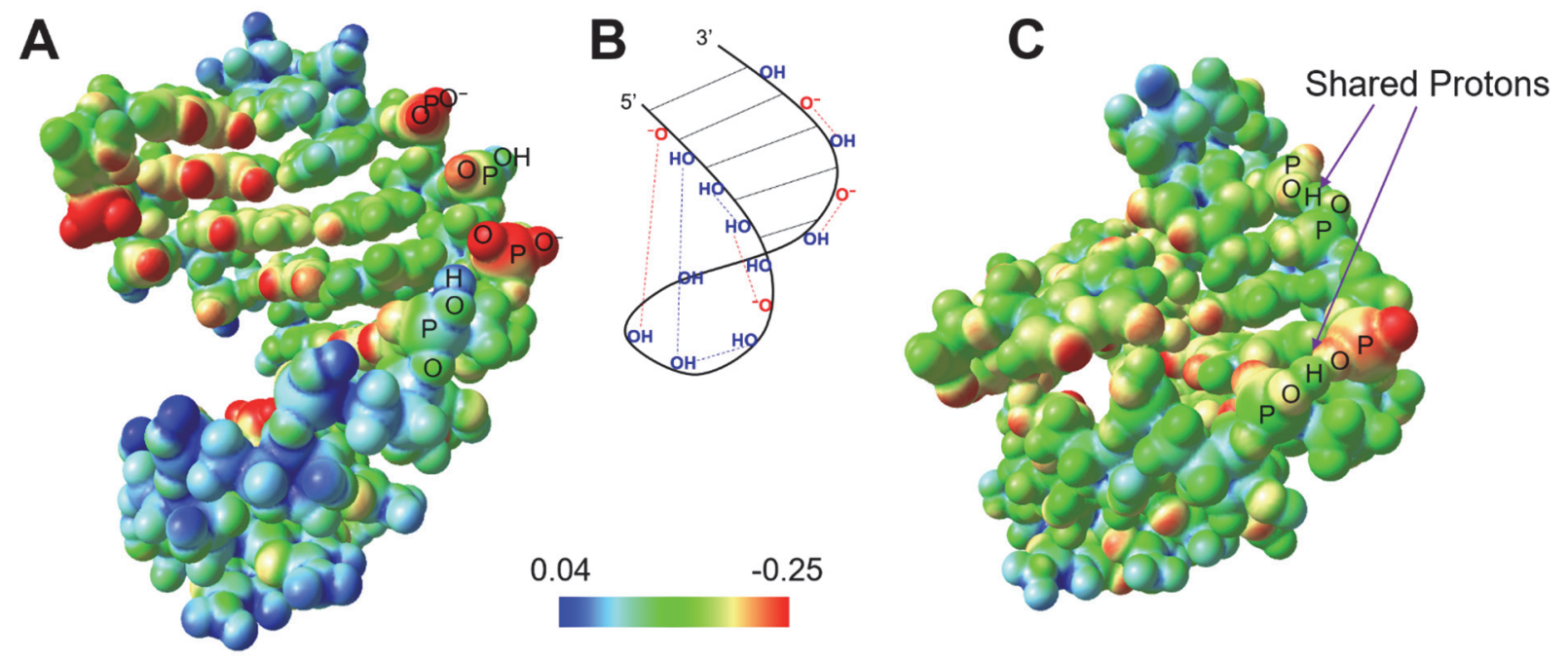

Figure 5: Starting structure (A) and DFT-optimized structure $(C)$ of the hairpin TAR ${ }^{4-}$. The color coding represents the electrostatic potential calculated from the total electronic density (blue: positive charges; red: negative charges). Scheme (B) shows the pattern of formation of new (non-native) hydrogen bonds among phosphate groups during optimization. 


\section{Conclusions and perspectives:}

It is usually believed that using the lowest charge states possible to minimize Coulomb repulsion is a good strategy to ensure a preservation of the solution structures into the gas phase. Here we showed that small RNA structures such as hairpins and kissing complexes rearrange when passing from the solution to the gas phase, and become more compact at the charge states obtained from $150 \mathrm{mM} \mathrm{NH}{ }_{4} \mathrm{OAc}$. Adding $\mathrm{Mg}^{2+}$ at concentrations that still allow to detect the kissing complexes did not rescue the structure. These results echo those found previously for DNA and RNA double helices. ${ }^{11}$ It does not necessarily mean that all aspects of the solution structure are lost. High-level structure optimization shows that base pairs are preserved while backbone rearrangements are responsible for a significant compaction. The formation of new (non-native) hydrogen bonds between phosphate groups has to be taken into account for the structural interpretation of ion mobility results.

Clearly, rearrangements occurring for low charge states are still hard to fully grasp and thus to predict. As a perspective, we propose two complementary research directions. First, on the experimental point of view, we have to explore what drives the adoption of a given charge state in the first place, given that the charge state influences the gas-phase structure (too low, it can condense by formation of non-native intramolecular bonds; too high, it can extend by breaking the native ones). Second, on the modeling point of view, we suggest that, instead of force field molecular dynamics simulations, higher-level calculations are required, ideally Car-Parrinello ${ }^{50}$ or Born-Oppenheimer molecular dynamics. ${ }^{39}$ We also anticipate that different starting geometries and different starting proton locations will lead to different final modeled structures. The current limiting factor for using higher-level calculations to interpret ion mobility spectrometry data is computer power, but it is clear that this will become increasingly feasible. Developing force fields adapted to gas-phase calculations would also be worthwhile. ${ }^{8}$ However, a gas-phase nucleic acid force field would need to be polarizable, ${ }^{51}$ and the modeling procedure should account for mobile protons, as suggested for proteins. ${ }^{52-54}$ Meanwhile, we will explore how structure optimizations and molecular dynamics simulations with intermediate levels of accuracy, such as semi-empirical calculations, could perform to interpret collision cross section distributions. 
Acknowledgement: This work was funded by the European Research Council (grant ERC2013-CoG-616551-DNAFOLDIMS to VG).

\section{Associated content:}

Additional experimental and computational results as described in text (PDF).

\section{$\underline{\text { References: }}$}

1. Gabelica, V.; Marklund, E., Fundamentals of Ion Mobility Spectrometry. Curr. Opin. Chem. Biol. 2018, 42, 51-59.

2. $\quad$ Kanu, A. B.; Dwivedi, P.; Tam, M.; Matz, L.; Hill, H. H., Ion mobility-mass spectrometry. J. Mass Spectrom. 2008, 43 (1), 1-22.

3. Rolland, A. D.; Prell, J. S., Computational insights into compaction of gas-phase protein and protein complex ions in native ion mobility-mass spectrometry. Trends Anal. Chem. 2019, $116,282-291$.

4. Jhingree, J. R.; Bellina, B.; Pacholarz, K. J.; Barran, P. E., Charge Mediated Compaction and Rearrangement of Gas-Phase Proteins: A Case Study Considering Two Proteins at Opposing Ends of the Structure-Disorder Continuum. J. Am. Soc. Mass Spectrom. 2017, 28 (7), 1450-1461. 5. Hoaglund, C. S.; Counterman, A. E.; Clemmer, D. E., Anhydrous protein ions. Chem. Rev. 1999, 99, 3037-3079.

6. Clemmer, D. E.; Russell, D. H.; Williams, E. R., Characterizing the Conformationome: Toward a Structural Understanding of the Proteome. Acc. Chem. Res. 2017, 50 (3), 556-560.

7. Pacholarz, K. J.; Porrini, M.; Garlish, R. A.; Burnley, R. J.; Taylor, R. J.; Henry, A. J.; Barran, P. E., Dynamics of intact immunoglobulin $\mathrm{G}$ explored by drift-tube ion-mobility mass spectrometry and molecular modeling. Angew. Chem. Int. Ed. 2014, 53 (30), 7765-9.

8. Allison, T.; Barran, P.; Cianferani, S.; Degiacomi, M.; Gabelica, V.; Grandori, R.; Marklund, E.; Menneteau, T.; Migas, L.; Politis, A.; Sharon, M.; Sobott, F.; Thalassinos, K.; Benesch, J. L., Computational Strategies and Challenges for Using Native Ion Mobility Mass Spectrometry in Biophysics and Structural Biology. Anal. Chem. 2020, 10.1021/acs.analchem.9b05791.

9. Gabelica, V.; De Pauw, E., Collision-Induced Dissociation of 16-mer DNA Duplexes with Various Sequences: Evidence for Conservation of the Double Helix Conformation in the Gas Phase. Int. J. Mass Spectrom. 2002, 219 151-159.

10. Gidden, J.; Baker, E. S.; Ferzoco, A.; Bowers, M. T., Structural motifs of DNA complexes in the gas phase. Int. J. Mass Spectrom. 2005, 240 (3), 183-193.

11. Porrini, M.; Rosu, F.; Rabin, C.; Darre, L.; Gomez, H.; Orozco, M.; Gabelica, V., Compaction of Duplex Nucleic Acids upon Native Electrospray Mass Spectrometry. ACS Cent. Sci. 2017, 3 (5), 454-461.

12. Baker, E. S.; Dupuis, N. F.; Bowers, M. T., DNA Hairpin, Pseudoknot, and Cruciform Stability in a Solvent-Free Environment. J. Phys. Chem. B 2009, 113 (6), 1722-1727. 
13. Lodish, H.; Berk, A.; Zipursky, S. L., Section 15.4, Intracellular Ion Environment and Membrane Electric Potential. In Molecular Cell Biology. 4th edition., W.H. Freeman: New York, 2000.

14. Nowakowski, J.; Tinoco, I., Jr., RNA Structure and Stability. Sem. Virol. 1997, 8, 153165.

15. Ladner, J. E.; Jack, A.; Robertus, J. D.; Brown, R. S.; Rhodes, D.; Clark, B. F. C.; Klug, A., Structure of yeast phenylalanine transfer RNA at 2.5 A resolution. Proc. Natl. Acad. Sci. USA 1975, 72 (11), 4414-4418.

16. Woese, C. R.; Winker, S.; Gutell, R. R., Architecture of ribosomal RNA: Constraints on the sequence of "tetra-loops" Proc. Natl. Acad. Sci. USA 1990, 87, 8467-8471.

17. Wedekind, J. E.; McKay, D. B., CRYSTALLOGRAPHIC STRUCTURES OF THE HAMMERHEAD RIBOZYME: Relationship to Ribozyme Folding and Catalysis. Annu. Rev. Biophys. Biomol. Struct. 1998, 27, 475-502.

18. Stephenson, W.; Asare-Okai, P. N.; Chen, A. A.; Keller, S.; Santiago, R.; Tenenbaum, S. A.; Garcia, A. E.; Fabris, D.; Li, P. T., The essential role of stacking adenines in a two-base-pair RNA kissing complex. J. Am. Chem. Soc. 2013, 135 (15), 5602-11.

19. Gregorian, R. S., Jr.; Crothers, D. M., Determinants of RNA Hairpin Loop-Loop Complex Stability. J. Mol. Biol. 1995, 248, 968-984.

20. Marino, J. P.; Gregorian, R. S., Jr.; Csankovszki, G.; Crothers, D. M., Bent Helix Formation Between RNA Hairpins with Complementary Loops. Science 1995, 268 (5216), 14481454.

21. Ducongé, F.; Toulmé, J.-J., In vitro selection identifies key determinants for loop-loop interactions: RNA aptamers selective for the TAR RNA element of HIV-1. RNA 1999, 5, 16051614.

22. Lee, A. J.; Crothers, D. M., The solution structure of an RNA loop-loop complex: the ColE1 inverted loop sequence. Structure 1998, 6, 993-1005.

23. Lebars, I.; Legrand, P.; Aime, A.; Pinaud, N.; Fribourg, S.; Di Primo, C., Exploring TAR-RNA aptamer loop-loop interaction by X-ray crystallography, UV spectroscopy and surface plasmon resonance. Nucleic Acids Res. 2008, 36 (22), 7146-56.

24. Gabelica, V.; Livet, S.; Rosu, F., Optimizing Native Ion Mobility Q-TOF in Helium and Nitrogen for Very Fragile Noncovalent Structures. J. Am. Soc. Mass Spectrom. 2018, 29 (11), 2189-2198.

25. Marchand, A.; Livet, S.; Rosu, F.; Gabelica, V., Drift Tube Ion Mobility: How to Reconstruct Collision Cross Section Distributions from Arrival Time Distributions? Anal. Chem. 2017, 89, 12674-12681.

26. D.A. Case, J. T. B., R.M. Betz, D.S. Cerutti, T.E. Cheatham, III, T.A. Darden, R.E. Duke, T.J. Giese, H. Gohlke, A.W. Goetz, N. Homeyer, S. Izadi, P. Janowski, J. Kaus, A. Kovalenko, T.S. Lee, S. LeGrand, P. Li, T. Luchko, R. Luo, B. Madej, K.M. Merz, G. Monard, P. Needham, H. Nguyen, H.T. Nguyen, I. Omelyan, A. Onufriev, D.R. Roe, A. Roitberg, R. Salomon-Ferrer, C.L. Simmerling, W. Smith, J. Swails, R.C. Walker, J. Wang, R.M. Wolf, X. Wu, D.M. York and P.A. Kollman, AMBER 2015. University of California, San Francisco. 2015.

27. Zgarbova, M.; Otyepka, M.; Sponer, J.; Mladek, A.; Banas, P.; Cheatham, T. E., 3rd; Jurecka, P., Refinement of the Cornell et al. Nucleic Acids Force Field Based on Reference Quantum Chemical Calculations of Glycosidic Torsion Profiles. J Chem Theory Comput 2011, 7 (9), 2886-2902. 
28. Perez, A.; Marchan, I.; Svozil, D.; Sponer, J.; Cheatham, T. E.; Laughton, C. A.; Orozco, M., Refinement of the AMBER force field for nucleic acids: improving the description of alpha/gamma conformers. Biophys. J. 2007, 92 (11), 3817-3829.

29. Banáš, P.; Mládek, A.; Otyepka, M.; Zgarbová, M.; Jurečka, P.; Svozil, D.; Lankaš, F.; Šponer, J., Can We Accurately Describe the Structure of Adenine Tracts in B-DNA? Reference Quantum-Chemical Computations Reveal Overstabilization of Stacking by Molecular Mechanics. J. Chem. Theory Comput. 2012, 8 (7), 2448-2460.

30. Jorgensen, W. L.; Chandrasekhar, J.; Madura, J. D.; Impey, R. W.; Klein, M. L., Comparison of simple potential functions for simulating liquid water. J. Chem. Phys. 1983, 79, 926-935.

31. Mahoney, M.; Jorgensen, W., A five-site model for liquid water and the reproduction of the density anomaly by rigid, nonpolarizable potential functions. J. Chem. Phys. 2000, 112 (20), 8910-8922.

32. Berendsen, H. J. C.; Postma, J. P. M.; van Gunsteren, W. F.; DiNola, A.; Haak, J. R., Molecular dynamics with coupling to an external bath. J. Chem. Phys. 1984, 81 (8), 3684-3690.

33. van Gunsteren, W. F.; Berendsen, H. J. C., Algorithms for macromolecular dynamics and constraint dynamics. Mol. Phys. 1977, 34 (5), 1311-1327.

34. Roe, D. R.; Cheatham, T. E., 3rd, PTRAJ and CPPTRAJ: Software for Processing and Analysis of Molecular Dynamics Trajectory Data. J. Chem. Theory Comput. 2013, 9 (7), 30843895.

35. $\mathrm{R}$ core team $R$ : A language and environment for statistical computing, $\mathrm{R}$ Foundation for Statistical Computing: Vienna, Austria, 2015.

36. Humphrey, W.; Dalke, A.; Schulten, K., VMD: visual molecular dynamics. J. Mol. Graph. 1996, 14 (1), 33-38.

37. Shvartsburg, A. A.; Mashkevich, S. V.; Baker, E. S.; Smith, R. D., Optimization of algorithms for ion mobility calculations. J. Phys. Chem. A 2007, 111 (10), 2002-2010.

38. Siu, C. K.; Guo, Y.; Saminathan, I. S.; Hopkinson, A. C.; Siu, K. W., Optimization of parameters used in algorithms of ion-mobility calculation for conformational analyses. J. Phys. Chem. B 2010, 114 (2), 1204-1212.

39. Swasey, S. M.; Rosu, F.; Copp, S. M.; Gabelica, V.; Gwinn, E. G., Parallel Guanine Duplex and Cytosine Duplex DNA with Uninterrupted Spines of Ag(I)-Mediated Base Pairs. $J$. Phys. Chem. Lett. 2018, 9 (22), 6605-6610.

40. D'Atri, V.; Porrini, M.; Rosu, F.; Gabelica, V., Linking molecular models with ion mobility experiments. Illustration with a rigid nucleic acid structure. J. Mass. Spectrom. 2015, 50 (5), 711-726.

41. Frisch, M. J.; Trucks, G. W.; Schlegel, H. B.; Scuseria, G. E.; Robb, M. A.; Cheeseman, J. R.; Scalmani, G.; Barone, V.; Mennucci, B.; Petersson, G. A.; Nakatsuji, H.; Caricato, M.; Li, X.; Hratchian, H. P.; Izmaylov, A. F.; Bloino, J.; Zheng, G.; Sonnenberg, J. L.; Hada, M.; Ehara, M.; Toyota, K.; Fukuda, R.; Hasegawa, J.; Ishida, M.; Nakajima, T.; Honda, Y.; Kitao, O.; Nakai, H.; Vreven, T.; Montgomery Jr., J. A.; Peralta, J. E.; Ogliaro, F.; Bearpark, M. J.; Heyd, J.; Brothers, E. N.; Kudin, K. N.; Staroverov, V. N.; Kobayashi, R.; Normand, J.; Raghavachari, K.; Rendell, A. P.; Burant, J. C.; Iyengar, S. S.; Tomasi, J.; Cossi, M.; Rega, N.; Millam, N. J.; Klene, M.; Knox, J. E.; Cross, J. B.; Bakken, V.; Adamo, C.; Jaramillo, J.; Gomperts, R.; Stratmann, R. E.; Yazyev, O.; Austin, A. J.; Cammi, R.; Pomelli, C.; Ochterski, J. W.; Martin, R. L.; Morokuma, K.; Zakrzewski, V. G.; Voth, G. A.; Salvador, P.; Dannenberg, J. J.; Dapprich, 
S.; Daniels, A. D.; Farkas, Ö.; Foresman, J. B.; Ortiz, J. V.; Cioslowski, J.; Fox, D. J. Gaussian 09, Gaussian, Inc.: Wallingford, CT, USA, 2009.

42. Zhao, Y.; Truhlar, D. G., The M06 suite of density functionals for main group thermochemistry, thermochemical kinetics, noncovalent interactions, excited states, and transition elements: two new functionals and systematic testing of four M06-class functionals and 12 other functionals. Theor. Chem. Acc. 2008, 120 (1-3), 215-241.

43. Grimme, S.; Antony, J.; Ehrlich, S.; Krieg, H., A consistent and accurate ab initio parametrization of density functional dispersion correction (DFT-D) for the 94 elements H-Pu. $J$. Chem. Phys. 2010, 132 (15), 154104.

44. Rabin, C. Investigation of RNA kissing complexes by native electrospray mass spectrometry : magnesium binding and ion mobility, $\mathrm{PhD}$ thesis, Université de Bordeaux, 2017. https://tel.archives-ouvertes.fr/tel-01717579.

45. Dong, S.; Wagner, N. D.; Russell, D. H., Collision-Induced Unfolding of Partially Metalated Metallothionein-2A: Tracking Unfolding Reactions of Gas-Phase Ions. Anal. Chem. 2018, 90 (20), 11856-11862.

46. Bartman, C. E.; Metwally, H.; Konermann, L., Effects of Multidentate Metal Interactions on the Structure of Collisionally Activated Proteins: Insights from Ion Mobility Spectrometry and Molecular Dynamics Simulations. Anal. Chem. 2016, 88 (13), 6905-13.

47. Kulesza, A.; Marklund, E. G.; MacAleese, L.; Chirot, F.; Dugourd, P., Bringing Molecular Dynamics and Ion-Mobility Spectrometry Closer Together: Shape Correlations, Structure-Based Predictors, and Dissociation. J. Phys. Chem. B 2018, 122 (35), 8317-8329. 48. Rueda, M.; Kalko, S. G.; Luque, F. J.; Orozco, M., The structure and dynamics of DNA in the gas phase. J. Am. Chem. Soc. 2003, 125, 8007-8014.

49. Hohenstein, E. G.; Chill, S. T.; Sherrill, C. D., Assessment of the Performance of the M05-2X and M06-2X Exchange-Correlation Functionals for Noncovalent Interactions in Biomolecules. J. Chem. Theory Comput. 2008, 4, 1996-2000.

50. Arcella, A.; Dreyer, J.; Ippoliti, E.; Ivani, I.; Portella, G.; Gabelica, V.; Carloni, P.; Orozco, M., Structure and dynamics of oligonucleotides in the gas phase. Angew. Chem. Int. Ed. 2015, 54 (2), 467-471.

51. $\quad$ Sponer, J.; Bussi, G.; Krepl, M.; Banas, P.; Bottaro, S.; Cunha, R. A.; Gil-Ley, A.; Pinamonti, G.; Poblete, S.; Jurecka, P.; Walter, N. G.; Otyepka, M., RNA Structural Dynamics As Captured by Molecular Simulations: A Comprehensive Overview. Chem. Rev. 2018, 118 (8), 4177-4338.

52. Fegan, S. K.; Thachuk, M., Controlling dissociation channels of gas-phase protein complexes using charge manipulation. J. Am. Soc. Mass Spectrom. 2014, 25 (5), 722-8.

53. Fegan, S. K.; Thachuk, M., A Charge Moving Algorithm for Molecular Dynamics Simulations of Gas-Phase Proteins. J. Chem. Theory Comput. 2013, 9 (6), 2531-9.

54. Popa, V.; Trecroce, D. A.; McAllister, R. G.; Konermann, L., Collision-Induced Dissociation of Electrosprayed Protein Complexes: An All-Atom Molecular Dynamics Model with Mobile Protons. J. Phys. Chem. B 2016, 120 (23), 5114-24. 
TOC graphics
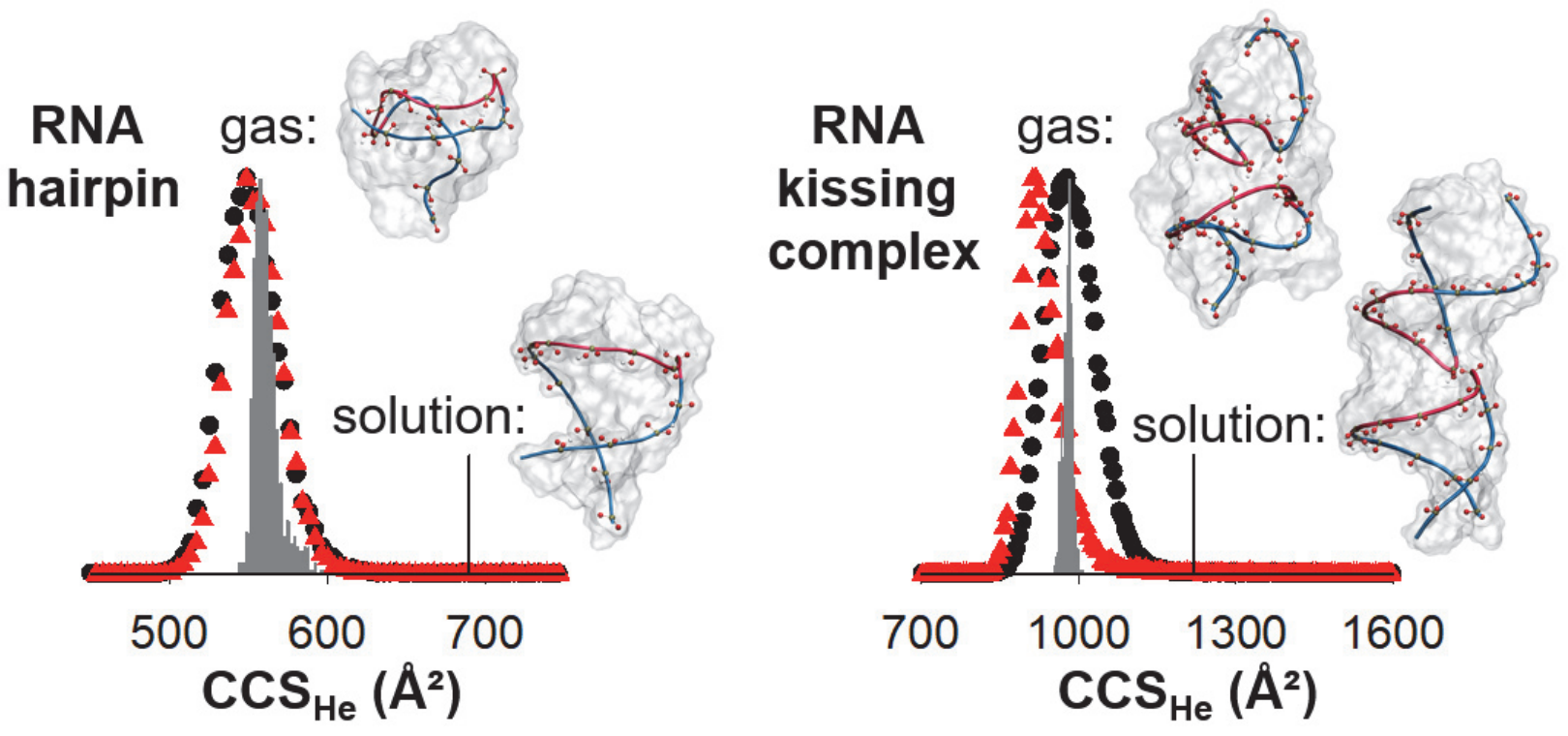